\title{
Curcumin Encapsulated into Methoxy Poly(Ethylene Glycol) Poly(ع-Caprolactone) Nanoparticles Increases Cellular Uptake and Neuroprotective Effect in Glioma Cells
}

Authors

Gregory Marslin ${ }^{1}$, Bruno Filipe Carmelino Cardoso Sarmento ${ }^{2,3}$, Gregory Franklin ${ }^{4}$, José Alberto Ribeiro Martins ${ }^{5}$, Carlos Jorge Ribeiro Silva $^{5}$, Andreia Ferreira Castro Gomes ${ }^{6}$, Marisa Passos Sárria ${ }^{6,7}$, Olga Maria Fernandes Pereira Coutinho ${ }^{1}$, Alberto Carlos Pires Dias ${ }^{1}$

Affiliations

1 Centre for the Research and Technology of Agro-Environment and Biological Sciences (CITAB-UM), AgroBioPlant Group, Department of Biology, University of Minho, Braga, Portugal

2 CESPU, IINFACTS - Instituto de Investigação e Formação Avançada em Ciências e Tecnologias da Saúde, Instituto Superior de Ciências da Saúde-Norte, Gandra-PRD, Portugal

3 INEB - Instituto de Engenharia Biomédica, University of Porto, Porto, Portugal

4 Department of Integrative Plant Biology, Institute of Plant Genetics, Polish Academy of Sciences, Poznań, Poland

5 Department of Chemistry, University of Minho, Braga, Portugal

6 CBMA - Centro de Biologia Molecular e Ambiental, Department of Biology, University of Minho, Braga, Portugal

7 INL - International Iberian Nanotechnology Laboratory, Braga, Portugal

Key words

curcumin, PEGylated nanoparticles, neuroprotection, cellular uptake, zebrafish

received Dec. 15, 2015

revised June 2, 2016

accepted July 2, 2016

Bibliography

DOI http://dx.doi.org/10.1055/s-0042-112030

Published online September 14, 2016 | Planta Med 2017; 83: 434-444

(c) Georg Thieme Verlag KG Stuttgart · New York I ISSN 0032-0943

Correspondence

Prof. Dr. Alberto Carlos Pires Dias

Centre for the Research and Technology of Agro-Environment and Biological Sciences (CITAB-UM), AgroBioPlant Group, Department of Biology, University of Minho

Campus de Gualtar, Braga 4710-057, Portugal

Phone: + 351910477281 , Fax: + 35125360319

acpdias@bio.uminho.pt

\begin{abstract}
Curcumin is a natural polyphenolic compound isolated from turmeric (Curcuma longa) with well-demonstrated neuroprotective and anticancer activities. Although curcumin is safe even at high doses in humans, it exhibits poor bioavailability, mainly due to poor absorption, fast metabolism, and rapid systemic elimination. To overcome these issues, several approaches, such as nanoparticle-mediated targeted delivery, have been undertaken with different degrees of success. The present study was conducted to compare the neuroprotective effect of curcumin encapsulated in poly( $\varepsilon$-caprolactone) and methoxy poly (ethylene glycol) poly( $\varepsilon$-caprolactone) nanoparticles in U251 glioblastoma cells. Prepared nanoparticles were physically characterized by laser doppler anemometry, transmission electron microscopy, and X-ray diffraction. The results from laser doppler anemometry confirmed that the size of poly( $\varepsilon$-caprolactone) and poly(ethylene glycol) poly( $\varepsilon$-caprolactone) nanoparticles ranged between $200-240 \mathrm{~nm}$ for poly( $\varepsilon$-caprolactone) nanoparticles and $30-70 \mathrm{~nm}$ for poly(ethylene glycol) poly ( $\varepsilon$-caprolactone) nanoparticles, and transmission electron microscopy images revealed their spherical shape. Treatment of U251 glioma cells and zebrafish embryos with poly( $\varepsilon$-caprolactone) and poly(ethylene glycol) poly( $\varepsilon$-caprolactone) nanoparticles loaded with curcumin revealed efficient cellular uptake. The cellular uptake of poly(ethylene glycol) poly( $\varepsilon$-caprolactone) nanoparticles was higher in comparison to poly( $\varepsilon$-caprolactone) nanoparticles. Moreover, poly(ethylene glycol) poly( $\varepsilon$-caprolactone) di-block copolymer-loaded curcumin nanoparticles were able to protect the glioma cells against tBHP induced-oxidative damage better than free curcumin. Together, our results show that curcumin-loaded poly(ethylene glycol) poly(e-caprolactone) diblock copolymer nanoparticles possess significantly stronger neuroprotective effect in U251 human glioma cells compared to free curcumin and curcumin-loaded poly( $\varepsilon$-caprolactone) nanoparticles.
\end{abstract}

\section{Introduction}

In human history, medicines derived from plants have played a critical role in the health care of many cultures [1]. Curcumin, a natural compound obtained from Curcuma longa has been used as a traditional therapeutic agent in Asian medicine to treat various common ailments [2]. Curcumin reportedly has multiple pharmacological effects, including anti-inflammatory, antioxidant, antibacterial, antivirus, and hyperlipidemic activities [3]. Although several studies have been conducted to explore the importance of curcumin, diverse issues restrict its clinical applica- 


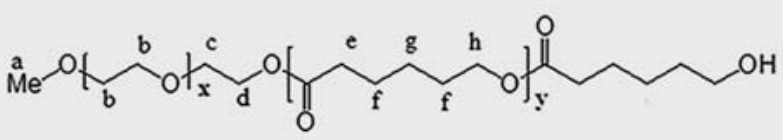

A

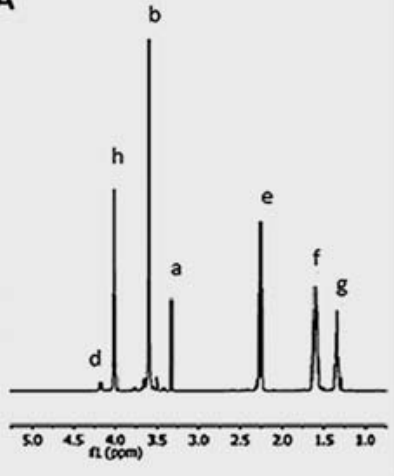

B

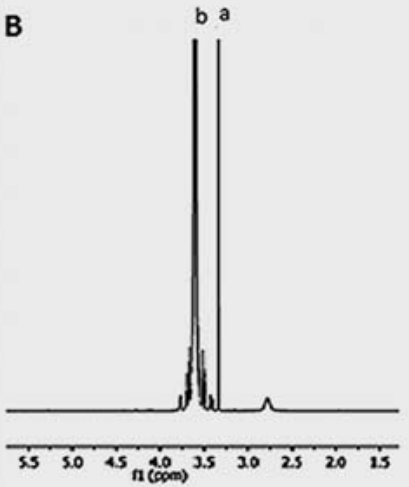<smiles>CC(CC(=O)OCCCCCC(=O)O)CC(C)CC(C)CC(C)C(C)C(=O)OCCCCCCO</smiles>

c

h e

Fig. 1 The ${ }^{1} \mathrm{H}$ NMR spectrum of the MPEG-PCL copolymer (A), MPEG (B), and PCL (C).

tion. The poor aqueous solubility of curcumin leads to its poor bioavailability and limits the in vivo pharmacokinetics, thereby restricting its effectiveness [4]. Aqueous solubility is a key parameter in drug development. More than half of new reactive molecular entities were not successfully developed into drugs because of their hydrophobicity [5]. So, it is important to obtain water-based formulations, with controlled release, for clinical application of curcumin [6].

Polymeric nanoparticles encapsulated with plant active compounds have been proven to be successful in increasing the bioavailability and retention time of some herbal medicines. For example, the neuroprotective effect of curcumin increased after converting it into a polymeric nanoformulation [7]. Likewise, Dhawan et al. demonstrated that solid lipid nanoparticles of quercetin delivered the drug to the brain and have a greater potential to treat Alzheimer's disease [8].

Methoxy poly(ethylene glycol) poly( $\varepsilon$-caprolactone) (MPEG$\mathrm{PCL}$ ) is an amphilic di-block copolymer composed of both hydrophilic and hydrophobic segments, which are capable of self-assembling into nanoscale spherical structures with a hydrophilic outer shell and a hydrophobic inner core [9]. The lipophilic drugs can be incorporated into the hydrophobic core of the nanosphere, while its hydrophilic outer shell still exists as a stabilizer for the system. The core-shell structure with poly(ethylene glycol) (PEG) as an outer shell enables the nanoparticles to escape from the scavenging of the mononuclear phagocyte system (MPS) effectively after systemic administration [10]. To enhance the water solubility, cellular uptake, and protective effect of curcumin, we have developed curcumin-loaded poly( $\varepsilon$-caprolactone) (PCL) and MPEG-PCL nanoparticles and evaluated their cellular uptake and neuroprotective activity in U251 human glioblastoma cells. Additionally, in vivo distribution of these nanoparticles was further investigated using zebrafish embryos.

\section{Results and Discussion}

Previous work by Deng and co-workers demonstrated that the molecular weight of MPEG-PCL copolymers displaying sharp unimodal distributions could be tuned by adjusting the molar ratio MPEG/PCL [11]. The polymers synthesized in this work were characterized by ${ }^{1} \mathrm{H}$ NMR spectroscopy. Illustrative ${ }^{1} \mathrm{H}$ NMR spectra of the MPEG polymer, PCL homopolymer and block copolymer MPEG-PCL are shown in > Fig. 1 . All signals in the ${ }^{1} \mathrm{H}$ NMR spectrum of the MPEG-PCL copolymer ( $\boldsymbol{\vee}$ Fig. $\mathbf{1} \mathbf{A}$ ) can be attributed to the MPEC block [signals at dignal (d), 3.56 (b) ppm] and to the PCL block [signals at d $1.30 \mathrm{ppm}(\mathrm{g}), 1.65$ (f), 2.25 (e), and 4.10 (h) ppm], confirming the block nature of the synthesized polymer. In this work, we selected a feed ratio of $50 \%$ (weight) of MPEG for the synthesis of the MPEG-PCL copolymer. ${ }^{1} \mathrm{H}$ NMR analysis reveals that the copolymer displays an average molecular weight of around $12000 \mathrm{Da}$ (DPMPEG $=114$ and DPCL $~ 60$ ) as reported originally for MPEG-PCL polymers synthesized in similar conditions [11]. Gel permeation chromatography (GPC) analysis confirmed the presence of a single peak with a similar average molecular weight ( 12200 Da).

The Fourier transform infrared (FTIR) spectra for typical PCL and MPEG-PCL polymers are shown in > Fig. 2. The MPEG-PCL COpolymer clearly exhibited peaks characteristic of both MPEG and $\mathrm{PCL}$. The absorption band at $1727 \mathrm{~cm}^{-1}$ was attributed to the $\mathrm{C}=\mathrm{O}$ stretching vibration of $\varepsilon$-caprolactone. The absorption bands at $2890 \mathrm{~cm}^{-1}$ and $2946 \mathrm{~cm}^{-1}$ were attributed to the $\mathrm{C}-\mathrm{H}$ stretching vibration of MPEG-PCL and $\varepsilon$-caprolactone. The ${ }^{1} \mathrm{H}$ NMR and FTIR confirm the formation MPEG-PCL.

MPEG-PCL nanoparticles have great potential in drug delivery, as they are already successfully used to deliver several drugs [ 12 , 13]. It is well known that the attachment of PEG in nanoparticles enhances the circulation time and cellular uptake $[14,15]$. Curcumin is a hydrophobic agent. Interestingly, its encapsulation into 


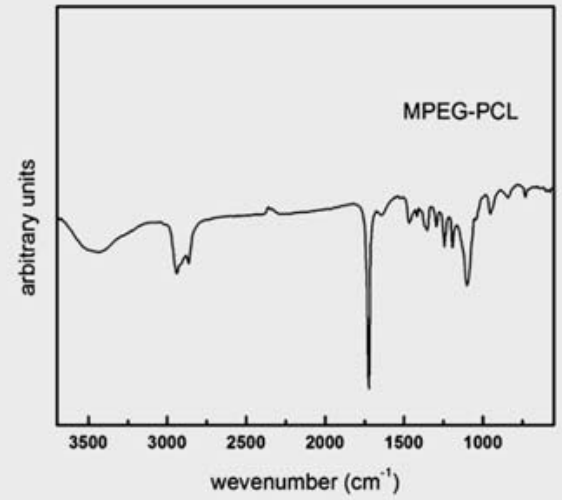

- Fig. 2 FTIR spectra of the MPEG-PCL copolymer.
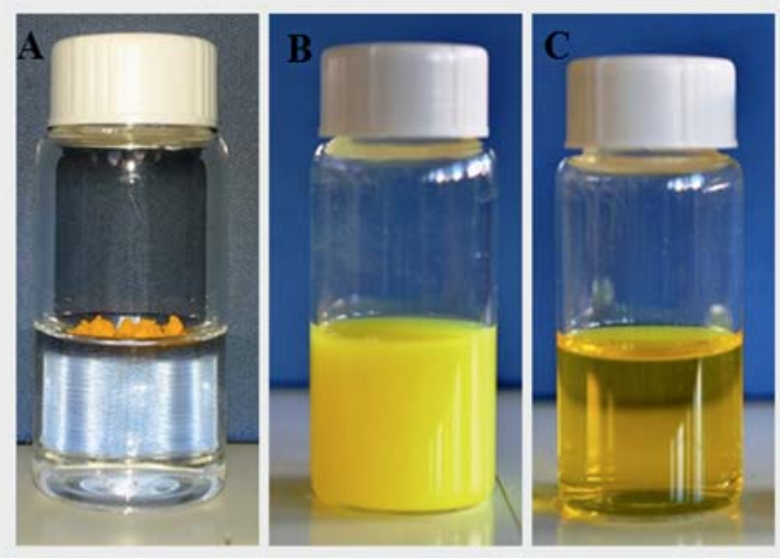

- Fig. 3 Aqueous solubility of free curcumin- (A), curcumin-PCL(B), and curcumin-MPEG-PCL-loaded (C) nanoparticles.

PCL or MPEG-PCL in the form of nanoparticles enhances the aqueous solubility of the compound ( $\bullet$ Fig. 3 ).

Poorly water-soluble drugs often require high doses in order to reach therapeutic plasma concentrations after oral administration. Low aqueous solubility is the major problem encountered in the formulation of new chemical entities, as well as generic drug development. Any drug, to be absorbed, must be present in the form of an aqueous solution at the site of absorption. However, most drugs are either weakly acidic or weakly basic, having poor aqueous solubility [16]. Since encapsulation of curcumin into $\mathrm{PCL}$ or MPEG-PCL nanoparticles increased their water solubility, we suggest that the usage of these nanoparticles may be a simple way to improve the absorption of some non-water soluble compounds.

The PCL and MPEG-PCL empty nanoparticles were characterized measuring their size, polydispersity (PDI), and $\zeta$-potential ( $\vee$ Table 1$)$. In general, empty PCL nanoparticles had a larger size and a more negative $\zeta$-potential when compared with their MPEG-PCL counterparts.
- Table 1 Size, PDI, and $\zeta$-potential of empty PCL and MPEG-PCL nanoparticles.

\begin{tabular}{|c|c|c|c|}
\hline $\begin{array}{l}\text { Formula- } \\
\text { tion code }\end{array}$ & $\begin{array}{l}\text { Size } \\
(n m \pm S D)\end{array}$ & $\begin{array}{l}\text { Polydispersity } \\
\text { (PDI } \pm \text { SD) }\end{array}$ & $\begin{array}{l}\text { \-Potential } \\
(\mathrm{mV} \pm \text { SD) }\end{array}$ \\
\hline EMP1 & $210.2 \pm 4.2$ & $0.21 \pm 0.24$ & $-24.6 \pm 2.2$ \\
\hline EMP2 & $226.1 \pm 4.6$ & $0.26 \pm 0.30$ & $-36.1 \pm 2.1$ \\
\hline EMP3 & $201.4 \pm 3.2$ & $0.32 \pm 0.24$ & $-31.1 \pm 3.1$ \\
\hline EMP4 & $204.3 \pm 3.3$ & $0.26 \pm 0.16$ & $-32.2 \pm 1.6$ \\
\hline EMP5 & $214.4 \pm 3.2$ & $0.20 \pm 0.26$ & $-27.1 \pm 1.8$ \\
\hline EMM1 & $54.3 \pm 3.6$ & $0.32 \pm 0.22$ & $-17.5 \pm 0.7$ \\
\hline EMM2 & $70.1 \pm 2.7$ & $0.36 \pm 0.15$ & $-15.3 \pm 1.2$ \\
\hline EMM3 & $36.4 \pm 1.6$ & $0.34 \pm 0.01$ & $-13.5 \pm 1.0$ \\
\hline EMM4 & $31.2 \pm 2.2$ & $0.32 \pm 0.03$ & $-9.3 \pm 1.4$ \\
\hline EMM5 & $48.6 \pm 2.4$ & $0.30 \pm 0.06$ & $-10.7 \pm 1.3$ \\
\hline \multicolumn{4}{|c|}{$\begin{array}{l}\text { Values are expressed as the mean } \pm \text { standard deviation }(n=3) \text {. EMP: } \\
\text { empty PCL nanoparticles; EMM: empty MPEG-PCL nanoparticles. These } \\
\text { nanoparticles were prepared accordingly the formulations indicated in } \\
\text { the Material and Methods section ( } \triangleright \text { Table } 3 \text { ), but without curcumin }\end{array}$} \\
\hline
\end{tabular}

Curcumin encapsulated PCL and MPEG-PCL nanoparticles were prepared by a solvent displacement method. Curcumin and MPEG-PCL were first dissolved in acetone. Then, this organic phase was added to an aqueous phase containing a surfactant. In the structure of MPEG-PCL, PEG is the hydrophilic segment and PCL is the hydrophobic segment, so the MPEG-PCL micelles always had a core-shell structure with a PCL core and a PEG shell. Self-assembly of curcumin and MPEG-PCL created a core-shell. It is well known that curcumin is not soluble in aqueous medium, but the prepared nanoparticles showed better aqueous solubility ( $\vee$ Fig. 3). The solubility of free curcumin in PBS was much less $(<0.5 \mathrm{mg} / \mathrm{L})$ when compared with the solubility of curcumin encapsulated in PCL (1.1 g/L) or in MPEG-PCL ( $2.9 \mathrm{~g} / \mathrm{L})$.

The size distribution of curcumin-loaded PCL and MPEG-PCL nanoparticles were measured by Dynamic Light Scattering (DLS) and the size distribution of the nanoparticles is shown in $\mathbf{F i g .} \mathbf{4}$. The average size of the nanoparticles loaded with curcumin ranged between $226-250 \mathrm{~nm}$ for PCL nanoparticles, whereas their MPEG-PCL nanoformulations produced much smaller particles in the size range of $44-72 \mathrm{~nm}$ ( $\bullet$ Table 2 ). A similar trend was also observed for the empty nanoparticles ( $\bullet$ Table 1 ). In general, nanoparticles with curcumin had a slightly, but significantly higher, size and had a more negative $\zeta$-potential compared to the empty ones, evidencing the incorporation of curcumin into the nanoparticles.

$\mathrm{PDI}$ and $\zeta$-potential of the nanoparticles are important physicochemical parameters that influence the stability of the polymers. The curcumin-loaded MPEG-PCL nanoparticles CURM4 had a PDI of approximately 0.22 , exhibiting a homogeneous distribution in diameter $(\triangleright$ Table 2$)$. Moreover, the lower $\zeta$-potential value $(-13.5 \mathrm{mV})$ of these nanoparticles further enhances their suitability compared to other batches. Additionally, this formulation was the one showing the highest entrapment efficiency of curcumin (circa 86\%). For curcumin-loaded PCL nanoparticles, the formula- 
A

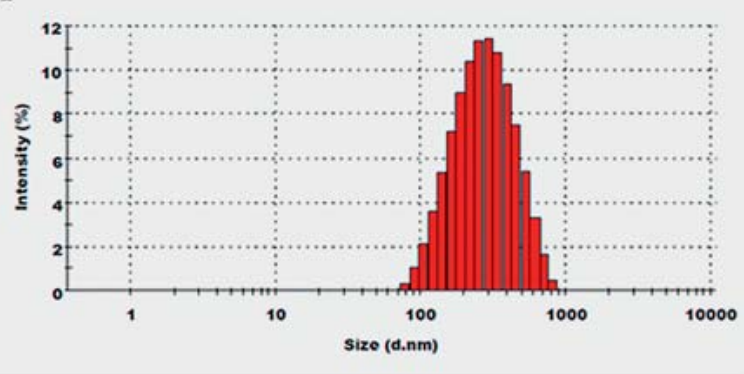

B

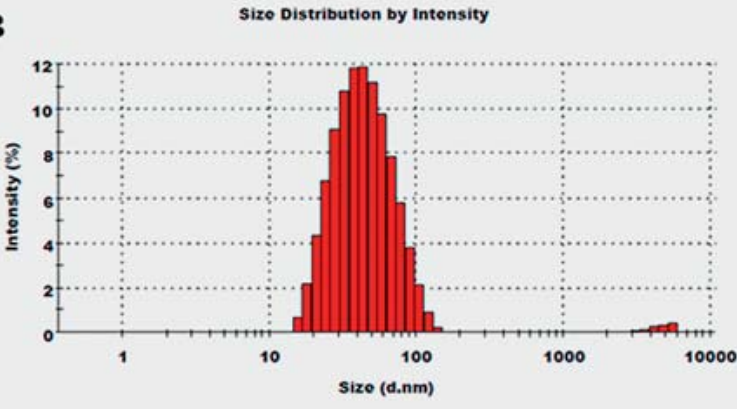

- Fig. 4 Characterization of curcumin-loaded PCL and MPEG-PCL nanoparticles. Size distribution of curcumin-PCL nanoparticles (A) and of curcumin-MPEG-PCL nanoparticles (B).

tion PCL4 was the one evidencing the highest entrapment efficiency of curcumin (circa 72\%), with good size and PDI values. Taking this into consideration, namely, the entrapment efficiency, the nanoparticles produced using PCL4 and MPCL4 formulations were the ones selected for the studies with U251 cells and zebrafish.

The morphology of the nanoparticles was determined by transmission electron microscopy (TEM). The obtained images showed that both curcumin-loaded PCL and MPEG-PCL nanoparticles ( $\bullet$ Fig. 5 A, B) have a smooth spherical shape and size ranging from $200-250 \mathrm{~nm}$ for PCL and 30-70 nm for MPEG-PCL nanoparticles. The results obtained regarding particle size in DLS correlate with the results obtained with TEM.

The release profile of free curcumin and curcumin-loaded PCL and MPEG-PCL nanoparticles was studied in vitro ( $\bullet$ Fig. 6 ). In the case of the free compound, approximately $70 \%$ of curcumin was released to the media in the first $24 \mathrm{~h}$. For the encapsulated curcumin, and in the same period, only $36 \%$ or $44 \%$ was released from the PCL or MPEG-PCL nanoparticles, respectively. Both the PCL and MPEG-PCL nanoparticles exhibited an initial burst release followed by a slower sustained release pattern. The initial burst release might be attributed to the diffusion of curcumin from PCL or MPEG-PCL nanoparticles. Additionally, curcumin MPEG-PCL nanoparticles attained a more sustained release compared with $\mathrm{PCL}$ nanoparticles. The controlled release pattern of loaded curcumin PCL and MPEG-PCL nanoparticles might help to achieve better pharmacological dynamics and kinetics.

Differential scanning calorimetry (DSC) analysis was performed to determine the physical nature of the drug in the nano- particles ( $\bullet$ Fig. 7). The DSC is an accurate and rapid thermo analytical technique widely used by the pharmaceutical industry and in drug research to investigate several physicochemical phenomena, such as polymorphism, melting and crystallization, purity, drug-excipient interaction, and polymer properties. Free curcumin had an endothermic melting peak at $178.59^{\circ} \mathrm{C}$, implying its crystalline state [17]. This peak was absent in the thermogram of curcumin-loaded PCL and MPEG-PCL nanoparticles, which only exhibited the characteristic melting peaks of MPEG-PCL around $55.52^{\circ} \mathrm{C}$.

The absence of detectable crystalline domains of curcumin in the loaded nanoparticles indicates that encapsulated curcumin was in the amorphous form in the polymeric matrix. The presence of drugs in crystalline that form inside the nanoparticles can be an obstacle due to the difficulty in the diffusion of large-sized molecules from the small pores. However, a sustained release of the encapsulated drug is possible if the drug is in the amorphous phase, as it facilitates easy diffusion of drug molecules through the polymeric matrix.

To examine the crystallinity of nano-curcumin, $X$-ray diffraction (XRD) analysis was carried out. XRD studies on pure curcumin, MPEG-PCL, and curcumin-loaded PCL and MPEG-PCL nanoparticles were performed using an $\mathrm{X}$-ray diffractometer. MPEG-PCL exhibited diffraction peaks at $2 \theta$ values of $19.2^{\circ}, 21.4^{\circ}$ and $23.5^{\circ}$ ( $\triangleright$ Fig. 8 ).

By comparing the XRD patterns of free curcumin and curcumin-loaded nanoparticles, it was found that the characteristic crystalline peaks of free curcumin were absent in the analysis of curcumin-loaded PCL and MPEG-PCL nanoparticles. This suggests that curcumin was molecularly dispersed and no curcumin crystals were formed once encapsulated in the nanoparticles, which permits the conclusion that the encapsulated curcumin was in the amorphous state.

The MTT colorimetric assay is a well-established method for determining the cytotoxic effect of substances at the level of cellular metabolism [18]. Since it is important to know whether an eventual cytotoxic effect is induced by the polymer or the drug, first we studied the cytotoxicity of empty nanoparticles at different concentrations $(1,10,50,100,500 \mu \mathrm{g} / \mathrm{mL})$. As shown in $>$ Fig. 9, it is evident that the empty PCL and MPEG-PCL nanoparticles were not significantly toxic up to $500 \mu \mathrm{g} / \mathrm{mL}$. It should be noted that $\mathrm{PCL}$ is a nontoxic, biodegradable, and biocompatible polymer and, therefore, approved by U. S. Food and Drug Administration (FDA) [12]. However, this polymer shows a slow degradation rate in vivo. MPEG-PCL has been used since it has some advantages, including stabilization of the delivery vehicle against undesirable aggregation and electrostatic interactions, whereas the PEG domain also increases the circulatory half-life in the body [12].

Cytotoxicity of free curcumin was also tested by the MTT assay. The obtained results showed that free curcumin inhibited cell viability in a concentration-dependent manner. From the concentrations screened, $25 \mu \mathrm{g} / \mathrm{mL}$ of free curcumin showed a significant reduction in cell viability compared to the control ( $\triangleright$ Fig. 10). Approximately $50 \%$ inhibition of cell viability was observed at the $25 \mu \mathrm{g} / \mathrm{mL}$ concentration of curcumin delivered in the form of PCL- and MPEG-PCL-encapsulated nanoparticles, similarly with that observed for free curcumin ( $\bullet$ Fig. 11). Moreover, a signifi- 
- Table 2 Size, PDI, $\zeta$-potential, and entrapment efficiency of curcumin-loaded PCL and MPEG-PCL nanoparticles. Values are expressed as the mean \pm standard deviation $(n=5)$.

\begin{tabular}{|l|l|l|l|l|}
\hline Formulation code & Size $(\mathbf{n m} \pm \mathbf{S D})$ & Polydispersity $(\mathbf{P D I} \pm \mathbf{S D})$ & $\zeta$-Potential $(\mathbf{m V} \pm \mathbf{S D})$ & Entrapment efficiency $(\% \pm$ SD) \\
\hline PCL1 & $250.2 \pm 2.4$ & $0.32 \pm 0.14$ & $-14.6 \pm 2.2$ & $39.1 \pm 2.1$ \\
\hline PCL2 & $243.2 \pm 3.2$ & $0.28 \pm 0.20$ & $-26.1 \pm 2.1$ & $46.1 \pm 3.5$ \\
\hline PCL3 & $232.6 \pm 2.6$ & $0.32 \pm 0.34$ & $-14.1 \pm 3.2$ & $42.2 \pm 2.3$ \\
\hline PCL4 & $224.3 \pm 2.3$ & $0.24 \pm 0.26$ & $-22.2 \pm 1.6$ & $72.3 \pm 3.3$ \\
\hline PCL5 & $226.5 \pm 5.2$ & $0.18 \pm 0.16$ & $-17.1 \pm 1.8$ & $46.3 \pm 2.2$ \\
\hline MPCL1 & $66.4 \pm 2.7$ & $0.22 \pm 0.24$ & $-16.4 \pm 0.5$ & $38.1 \pm 3.4$ \\
\hline MPCL2 & $72.2 \pm 3.1$ & $0.26 \pm 0.14$ & $-13.2 \pm 1.3$ & $42.1 \pm 2.3$ \\
\hline MPCL3 & $44.2 \pm 1.5$ & $0.24 \pm 0.09$ & $-11.6 \pm 2.4$ & $56.6 \pm 4.1$ \\
\hline MPCL4 & $46.2 \pm 2.3$ & $0.22 \pm 0.01$ & $-13.5 \pm 2.1$ & $85.8 \pm 1.8$ \\
\hline MPCL5 & $56.3 \pm 3.1$ & $0.20 \pm 0.06$ & $-13.6 \pm 1.1$ & $59.2 \pm 2.1$ \\
\hline PCL: Curcumin-loaded PCL nanoparticles; MPCL: curcumin-loaded MPEG-PCL nanoparticles. & \\
\hline
\end{tabular}

cant decrease in cell viability (circa $25 \%$ ) was observed with $10 \mu \mathrm{g} /$ $\mathrm{mL}$ of curcumin nanoencapsulated, which was not observed for free curcumin. When considering lower concentrations of curcumin-loaded nanoparticles $(0.01,0.1$, and $1 \mu \mathrm{g} / \mathrm{mL})$, the numbers of viable cells were similar to the control ( $\triangleright$ Fig. 11). Hence, 0.1 , 1 , and $10 \mu \mathrm{g} / \mathrm{mL}$ of curcumin-encapsulated PCL and MPEG-PCL nanoparticles were chosen for further neuroprotection assays.

Curcumin is an autofluorescent compound that emits green fluorescence when viewed under a $535-600 \mathrm{~nm}$ filter [19]. Following 4,6 , and $24 \mathrm{~h}$ exposure of U251 cells with $1 \mu \mathrm{g} / \mathrm{mL}$ of free curcumin and curcumin-loaded PCL or MPEG-PCL nanoparticles, the green fluorescence intensity increased in a time-dependent manner ( $\vee$ Fig. 12 A-C). Cells with free curcumin showed the lowest or no fluorescence. Maximum fluorescence intensity for each treatment was observed in cells that underwent $24 \mathrm{~h}$ coincubation. MPEG-PCL nanoparticle-treated cells showed the highest intensity of fluorescence compared to $\mathrm{PCL}$ nanoparticles ( $\vee$ Fig. 12A-C), clearly revealing the importance of MPEG conjugation for the highest cellular uptake of nanoparticles.

While cells incubated with free curcumin failed to register any fluorescence even after $24 \mathrm{~h}$, the cells treated with curcuminloaded nanoparticles showed fluorescence, indicating that nanoformulation improves the cellular uptake of curcumin. Among PCL and MPEG-PCL curcumin-loaded nanoparticles, the later produced a significantly higher fluorescence in cells than curcuminPCL nanoparticles. Interestingly, the fluorescence signal displayed by curcumin-PCL nanoparticles was more superficial and weaker than the one generated by curcumin-MPEG-PCL nanoparticles, suggesting that the superficial signal might be from the nanoparticles attached to the surface of the cells, and the lighter intensity could be due to curcumin dispersion into the cytoplasm rather than accumulated in the nuclei. On the other hand, cells treated with curcumin-MPEG-PCL nanoparticles showed fluorescence, not only in the cytoplasm but also in the nuclei, overall increasing the intensity of fluorescence. Since MPEG-PCL nanoparticles had a stronger fluorescence intensity, we can conclude that the attachment of MPEG to PCL has increased the cellular uptake of the nanoparticles. Overall, curcumin vehiculated in the form of
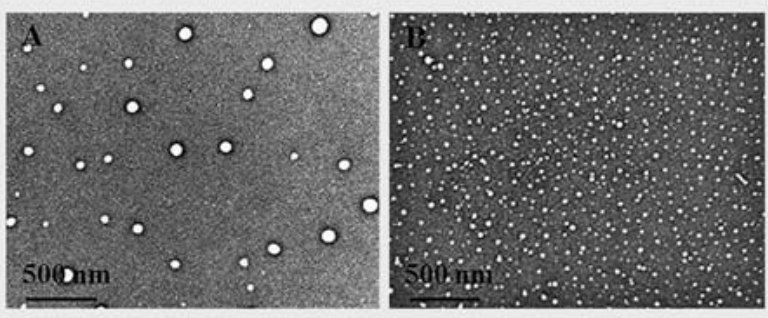

- Fig. 5 Morphological characterization by TEM of curcuminloaded PCL nanoparticles (A) and MPEG-PCL nanoparticles (B).

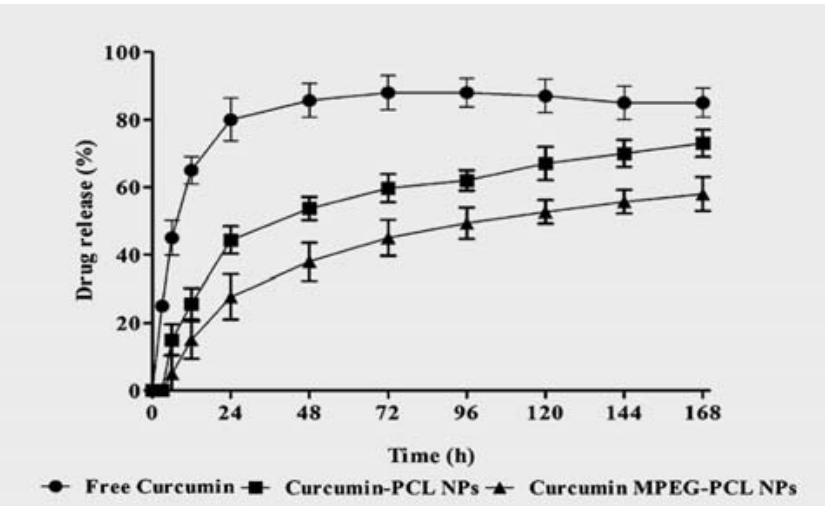

- Fig. 6 In vitro drug release profile of curcumin from PCL and MPEG-PCL nanoparticles (NPs).

MPEG-PCL nanoparticles was an efficient way to penetrate cells and get into the cytoplasm and nucleus.

The neuroprotective effect of free curcumin and curcuminloaded PCL and MPEG-PCL nanoparticles was analyzed in U-251 cells submitted to oxidative stress with $1 \mathrm{mM}$ of tert-butyl hydro- 


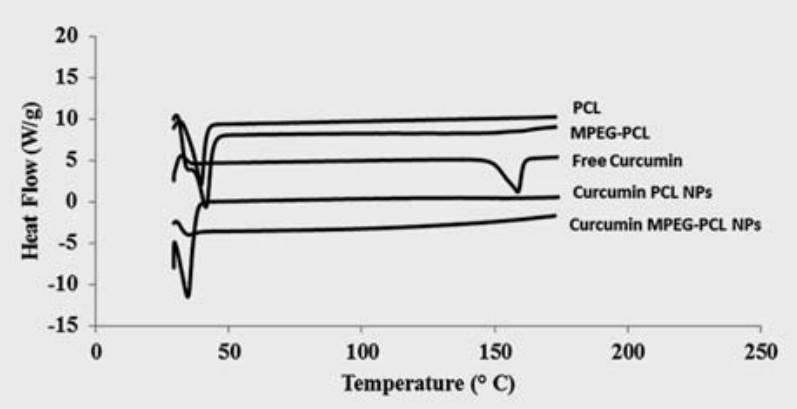

- Fig. 7 Differential scanning calorimetric thermogram of loadedcurcumin PCL and MPEG-PCL nanoparticles (NPs).

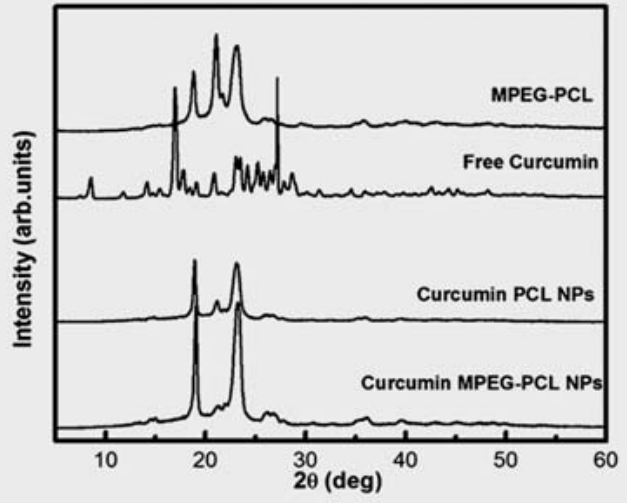

- Fig. 8 X-ray diffraction patterns of MPEG-PCL, free curcumin, and curcumin-loaded PCL and MPEG-PCL nanoparticles (NPS).

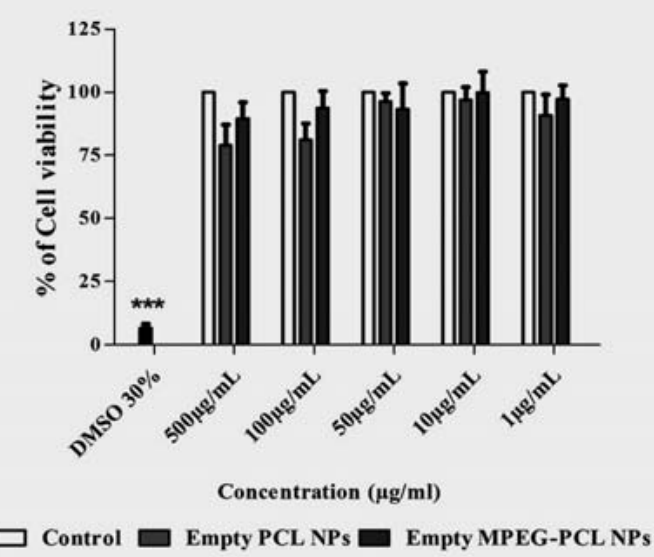

- Fig. 9 In vitro cytotoxicity of the empty PCL and MPEG-PCL nanoparticles (NPs). Data represents the mean \pm SD of three independent sets of experiments (total $n=15$ ). No statistical differences were found among experiments. DMSO at a concentration of $30 \%$ (in cell's medium) was used as a positive control for the cytotoxic effect. peroxide (t-BHP) for $3 \mathrm{~h}$. In this condition, the cells showed less than $10 \%$ of viability when compared with cells in a non-insult condition (control cells).

Three different concentrations of free curcumin $(0.1,1$, and $10 \mu \mathrm{g} / \mathrm{mL}$ ), empty PCL and MPEG-PCL nanoparticles, and curcumin-encapsulated PCL and MPEG-PCL nanoparticles were coincubated with the oxidant agent t-BHP. The empty PCL and MPEG-PCL nanoparticles did not show any protective effect when compared with the control (only cells). Nevertheless, free curcumin and curcumin-loaded PCL and MPEG-PCL nanoparticles showed significant neuroprotection against oxidative damage induced by t-BHP ( $\bullet$ Fig. 13 ). When compared with free curcumin, both types of curcumin-loaded nanoparticles led to a better protective effect for the same equivalent amount of curcumin. Curcumin encapsulated MPEG-PCL nanoparticles induced, however, a higher percentage of protection $(79.81 \%)$ for $0.1 \mu \mathrm{g} / \mathrm{mL}$, (84.57\%) for $1 \mu \mathrm{g} / \mathrm{mL}$, and (37.27\%) for $10 \mu \mathrm{g} / \mathrm{mL}$. The curcuminloaded PCL nanoparticles showed a less neuroprotective effect of $52.87 \%$ for $0.1 \mu \mathrm{g} / \mathrm{mL}, 38.63 \%$ for $1 \mu \mathrm{g} / \mathrm{mL}$, and $24.03 \%$ for $10 \mu \mathrm{g} /$ $\mathrm{mL}$. An interesting fact is that the neuroprotective effect of both the free and nanoformulated curcumin was reduced significantly at higher concentrations ( 1 and $10 \mu \mathrm{g} / \mathrm{mL}$ ) compared to $0.1 \mu \mathrm{g} /$ $\mathrm{mL}$ of curcumin, suggesting that this compound is more effective at low concentrations. In fact, we observed a cytotoxic effect in the cells for free curcumin and loaded-curcumin nanoparticles for higher concentrations ( $\triangleright$ Figs. 10 and $\mathbf{1 1}$ ).

Increased activity of free and encapsulated curcumin has been previously reported, although not for neuroprotection. For instance, it is known that the cellular uptake of curcumin improved when it was prepared as cationic chitosan/PCL nanoparticles [20]. Also, recently, curcumin was successfully encapsulated into polymeric nanoparticles and this nanoformulated curcumin showed better results than free curcumin against different cancer cell lines [21].

Curcumin encapsulated in (MPEG-)PCL nanoparticles provided neuroprotection against t-BHP-induced oxidative stress at very low concentrations when compared with free curcumin ( $\triangleright$ Fig. 13). Namely, this was observed for curcumin MPEG-PCL nanoparticles that showed a higher significant neuprotection for $0.1-1 \mu \mathrm{g} / \mathrm{mL}$ concentrations. The observed protective effect of curcumin nanoparticles may be due to the enhanced transport of curcumin into cells, and the sustainable release of compounds from the nanoparticles. This should have contributed to reaching an effective concentration of curcumin inside the cells and being able to avoid the negative oxidative effects of the t-BHP insult.

The in vivo distribution of curcumin in the free and nanoparticulate forms (PCL and MPEG-PCL) was further analyzed in a zebrafish model. No fluorescence was detected in 80 hpf zebrafish embryos treated with freshwater (control) and empty nanoparticles ( $\vee$ Fig. 14A-F). The zebrafish embryos treated with free curcumin and curcumin-loaded PCL nanoparticles showed fluorescence, although in a very low level ( $\bullet$ Fig. 14G-J). This was specially noticed for free curcumin, with zebra fish embryos showing a very faint and scarce fluorescence. However, the fluorescence intensity was significantly higher in the zebrafish embryos treated with curcumin-loaded MPEG-PCL nanoparticles compared to free curcumin and its PCL nanoformulation ( $\bullet$ Fig. $14 \mathrm{~K}-\mathrm{L}$ ). Moreover, as 


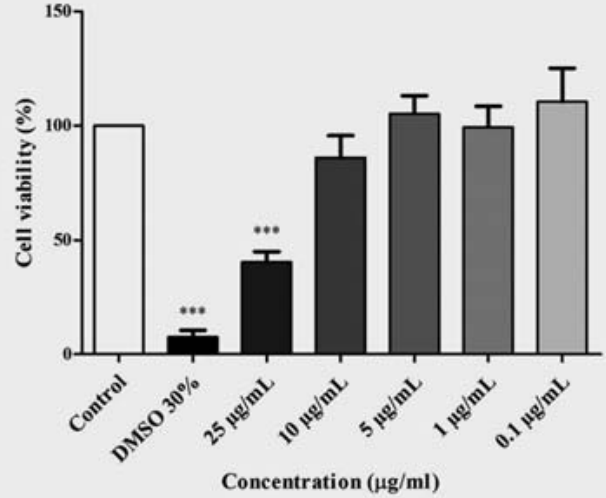

- Fig. 10 Cytotoxicity evaluation of free curcumin in U251 human glioma cells. Data represents the mean \pm SD of three independent sets of experiments (total $n=15$ ). Statistical analysis of the data was performed using a one sample t-test. Asterisks denote statistically significant differences from the control (cells without curcumin;

$*^{* * *} \mathrm{p}<0.001$ ). DMSO at a concentration of $30 \%$ (in cell's medium) was used as a positive control for the cytotoxic effect.

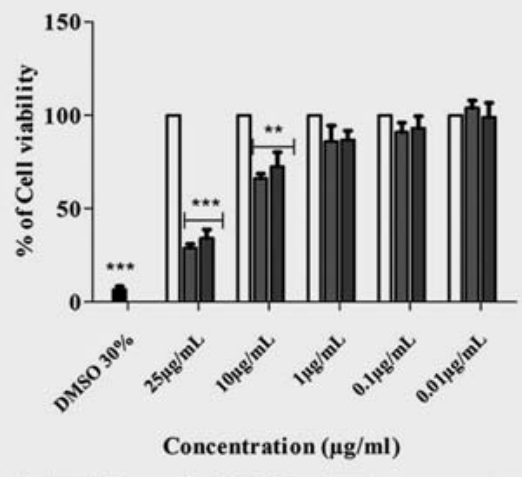

Control

Curcumin PCL NPs

Curcumin MPEG-PCL NPs

- Fig. 11 Cytotoxicity of curcumin-loaded PCL and MPEG-PCL nanoparticles (NPs) in U251 human glioma cells. Data represents the mean \pm SD of three independent sets of experiments (total $n=15$ ). Statistical analysis of the data was performed using a t-test. Asterisks denote statistically significant differences from the control (only cells; $\left.{ }^{*} p<0.05,{ }^{* *} p<0.01,{ }^{* * *} p<0.001\right)$. DMSO at a concentration of $30 \%$ (in medium) was used as a positive control for the cytotoxic effect.

seen in $>$ Fig. $14 \mathrm{~L}$, the curcumin-loaded MPEG-PCL nanoparticles were distributed throughout the zebrafish embryos. The nanoparticles reach mainly the yolk sac and other lipophilic areas, including the spinal cord. The preferential accumulation of free curcumin in zebrafish yolk was already reported before [22].

Accumulation of nanoparticles in the brain is of particular interest for brain delivery of drugs. Careful analysis revealed that the MPEG-PCL nanoparticles accumulated in the central nervous system in zebrafish embryos in a more intense way than PCL nanoparticles. Collectively, our results indicate that curcumin
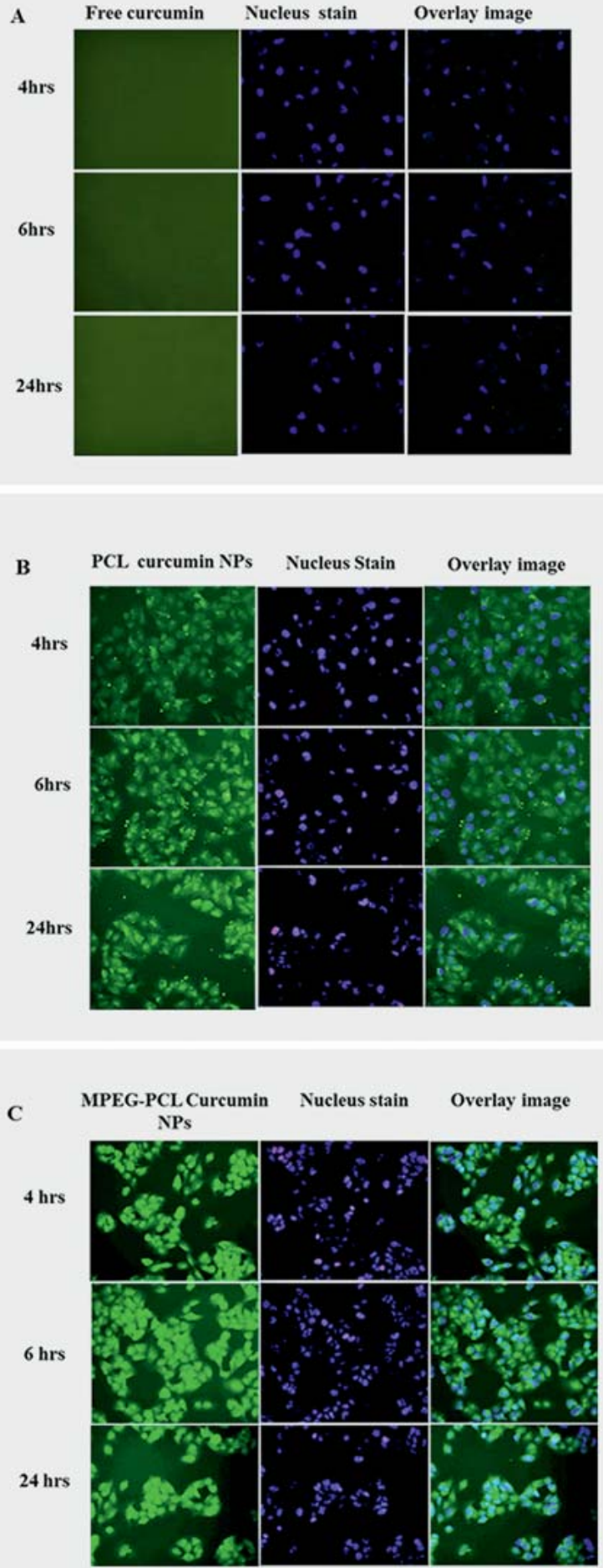

- Fig. 12 Human glioblastoma cells (U251) coincubated with $1 \mu \mathrm{g} /$ $\mathrm{mL}$ of free curcumin and curcumin-loaded PCL and MPEG-PCL nanoparticles (NPs) for different incubation times (4, 6, and $24 \mathrm{~h}$ ) were analyzed for their fluorescence intensity under a fluorescence microscope. Fluorescent microscope images of free curcumin (A), curcumin-loaded PCL nanoparticles (B), and curcumin-loaded MPEG-PCL nanoparticles (C) showing the internalization of nanoparticles by the U251 human glioma cells. 

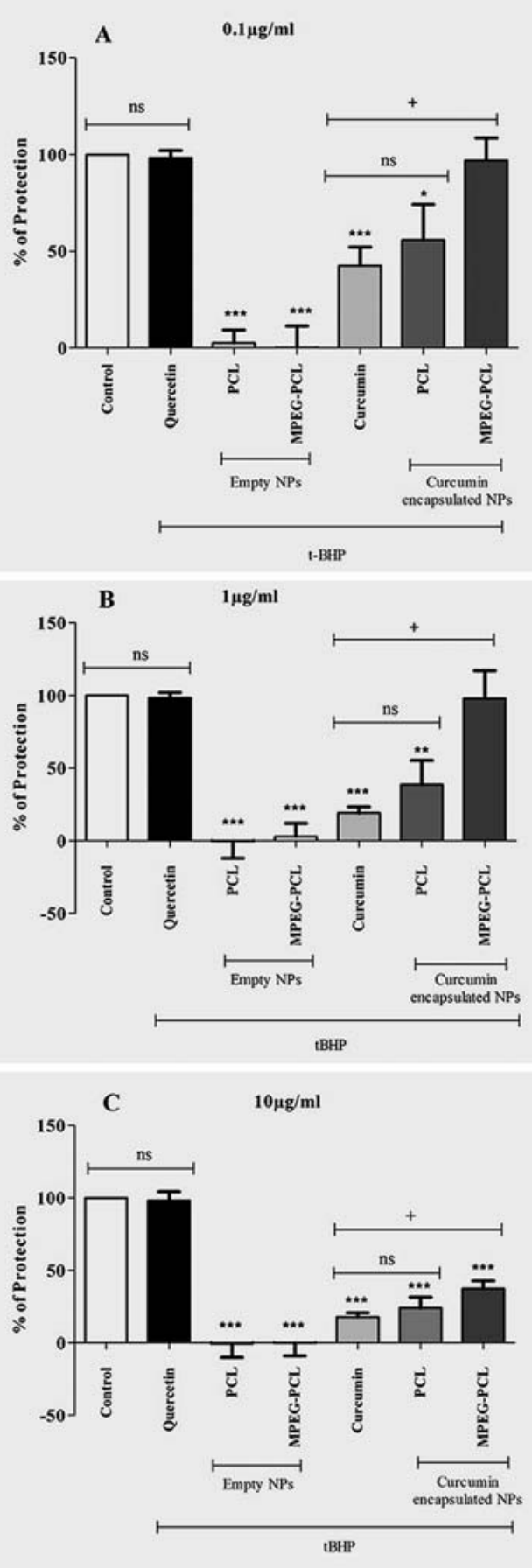

- Fig. 13 Neuroprotective effects of different concentrations of free curcumin and curcumin-loaded MPEG-PCL and PCL nanoparticles (NPs) $(0.1,1$, and $10 \mu \mathrm{g} / \mathrm{mL})$. Data represents the mean \pm SD of three independent experiments (total $n=15$ ). Statistical analysis of the data was performed using a t-test. Asterisks denote statistically significant differences from the control $\left({ }^{*} p<0.05,{ }^{*} p<0.01\right.$, $\left.{ }^{* * *} p<0.001\right)$; +denotes statistically significant differences between the columns $(p<0.05)$; ns = not significant. Control column was data from cells not exposed to the t-BHP insult; all other figures were from experiments with cells exposed to the t-BHP insult $(1 \mathrm{mM}, 3 \mathrm{~h})$. Quercetin $(10 \mu \mathrm{M})$ was used as a positive control for the neuroprotective effect against the t-BHP oxidative insult.
MPEG-PCL nanoencapsulation is highly efficient in terms of uptake by neural cells and zebra fish embryos. This is in accordance with previous reports of albumin-conjugated tanshinone IIA PEGylated nanoparticles, where PEGylation increased brain delivery of tanshinone IIA in rats [23].

Low water solubility of many compounds, like curcumin, reduces their bioavailability and stability. Moreover, is well known that the blood-brain barrier (BBB) is a major obstacle for molecules to reach the brain, reducing (or avoiding) the effectiveness of several neuroprotective compounds. To overcome these issues, the development of nanodelivery systems for neuroactive phytochemicals could be an interesting alternative [24]. In fact, in this work, we specifically used pluronic F-68 for the preparation of nanoparticles, a well-know and used surfactant that improves the solubility of low water-soluble compounds and the stability of colloidal interfaces. Additionally, F-68 enhances drug transport across the BBB and intestinal barriers [25], which is an advantage property for the present application.

In conclusion, in this work, a biodegradable PCL and MPEG-PCL polymer was synthesized to encapsulate curcumin. These nanostructures were characterized in size and shape. The cellular uptake of curcumin-loaded MPEG-PCL nanoparticles was higher when compared to free curcumin and curcumin encapsulated in PCL nanoparticles. Moreover, the MPEG-PCL nanoparticles showed a higher protective effect against oxidative damage induced by t-BHP in U251 glioma cells, and increased curcumin uptake both by cells and zebrafish embryos. Overall, the obtained results allow concluding that the MPEG-PCL nanoencapsulation of curcumin enhances its aqueous solubility, in vitro and in vivo uptake, and neuroprotective properties. This approach might have potential as an alternative for curcumin delivery, namely, for protecting nervous cells from oxidative damage.

\section{Materials and Methods}

\section{Cell line, chemicals, and biochemicals}

The U251 cell line (Human glioblastoma) was a gift from CESPU. Curcumin ( $\geq 94 \%$ curcuminoid content; $\geq 80 \%$ curcumin), quercetin ( $\geq 95 \%)$, stannous octoate, poly(ethylene glycol) methyl ether (MPEG, Mn 2000), dimethyl sulfoxide (DMSO, $\geq 99.9 \%$ ) and methylthiazolyldiphenyl-tetrazolium bromide (MTT) were purchased from Sigma-Aldrich. $\varepsilon$-Caprolactone $(\varepsilon-\mathrm{CL})$ was from Alfa Aesar, DMEM was from HyClone Thermo Scientific, and FBS was from BioChrom KG. All other solvents and reagents used in this study were ACS or HPLC analytical grade and were purchased from Merck.

\section{Synthesis and characterization of block copolymer methoxy poly(ethyleneglycol)-poly( $\varepsilon$-caprolactone) nanoparticles}

The MPEG-PCL block copolymer was prepared by ring-opening polymerization of monomethoxy-poly(ethylene glycol) and $\varepsilon$-caprolactone in the presence of stannous octoate as the catalyst, as described previously [24]. Briefly, dried (by molecular sieves) $\varepsilon$ $\mathrm{CL}(5 \mathrm{~g}, 43.8 \mathrm{mmol}$ ) and dried (by azeotropic distillation in toluene) MPEG $(5 \mathrm{~g}, 1 \mathrm{mmol})$ and stannous octoate catalyst $(0.1$ weight \%) were added into a flamed-dried and nitrogen-purged 


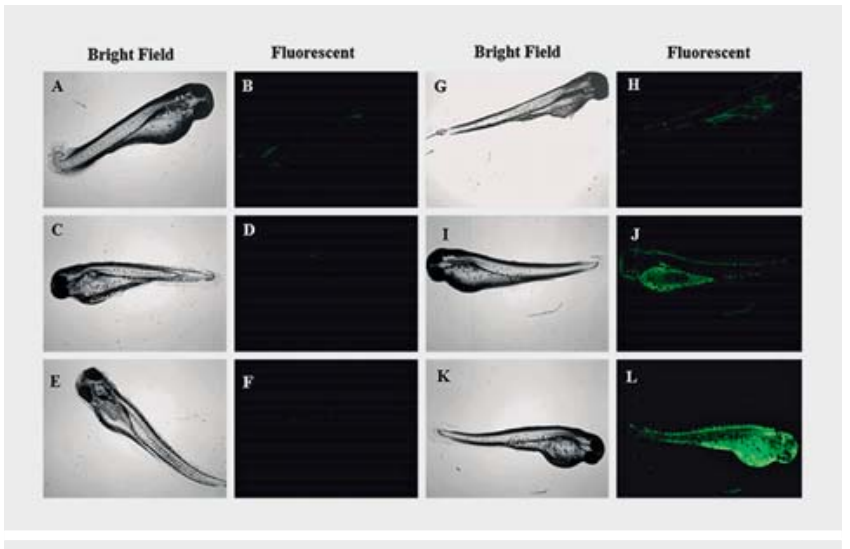

- Fig. 14 Accumulation of free curcumin and curcumin-loaded (MPEG-)PCL nanoparticles in 80 hpf zebrafish embryos. A-B Light and fluorescent microscopic views of control zebrafish. C-D Light and fluorescent microscopic views of zebra fish incubated with empty PCL nanoparticles. E-F Light and fluorescent microscopic views of zebrafish incubated with empty MPEG-PCL nanoparticles. G-H Light and fluorescent microscopic views of zebrafish incubated with free curcumin. I-J Light and fluorescent microscopic views of zebrafish incubated curcumin-loaded PCL nanoparticles. $\mathrm{K}-\mathrm{L}$ Light and fluorescent microscopic views of zebrafish incubated with curcumin-loaded MPEG-PCL nanoparticles.

50 - $\mathrm{mL}$ glass reactor. The reactor was sealed under reduced pressure $(5 \mathrm{mmHg})$ and the reaction was allowed to proceed at $140{ }^{\circ} \mathrm{C}$ for $24 \mathrm{~h}$. The reaction mixture was cooled down with water and diluted with $\mathrm{CHCl}_{3}$. The addition of a mixture of methanol: ether resulted in the precipitation of a white solid. The solid was filtered, washed with diethyl ether, and redissolved in $\mathrm{CHCl}_{3}$. The block copolymer was precipitated again by adding a mixture of methanol: diethyl ether, and then it was filtered and washed with diethyl ether. Drying under vacuum at $40^{\circ} \mathrm{C}$ for $48 \mathrm{~h}$ afford the copolymer in good yield (70\%). GPC of MPEG-PCL copolymer was performed in a Beckman Gold HPLC apparatus equipped with a refractive index detector and a Styragel-HR4E column (Waters), using tetrahydrofuran as the mobile phase. Polymers were characterized by relative elution time to polystyrene ReadyCal standards kit (Waters) in the range of 400 to $2000 \mathrm{kDa}$.

The copolymer was further characterized by FTIR and ${ }^{1} \mathrm{H}$ NMR. IR spectra were acquired on a Bomem MB104 193 spectrometer in the wave number range of $4000-500 \mathrm{~cm}^{-1}$ using the $\mathrm{KBr}$ pellet method ( $1 \mathrm{mg}$ of polymer dispersed in $200 \mathrm{mg}$ of $\mathrm{KBr}$ ). ${ }^{1} \mathrm{H}$ NMR spectra were acquired in deuterated chloroform at $25^{\circ} \mathrm{C}$ in a Brucker Avance III $400 \mathrm{MHz}$ spectrometer. Chemical shifts are reported in ppm with reference to residual chloroform, signal at $\mathrm{d}=7.27 \mathrm{ppm}$.

\section{Preparation of curcumin-encapsulated nanoparticles}

Curcumin encapsulation within the polymeric nanoparticles was achieved by the solvent displacement method. Briefly, $100 \mathrm{mg}$ of PCL or MPEG-PCL and $10 \mathrm{mg}$ of curcumin were dissolved in $5 \mathrm{~mL}$ of acetone with mild heating and sonication [11]. The acetone solution (organic phase) was introduced into $50 \mathrm{~mL}$ of aqueous solution containing $1 \%$ of Pluronic ${ }^{\circledR}$ (F-68) under magnetic stirring for
$4 \mathrm{~h}$. Residual acetone was removed from the suspension by evaporation at reduced pressure (rotary evaporator) at $37^{\circ} \mathrm{C}$. The resulting nanoparticle suspension was filtered through a syringe filter (pore size: $220 \mathrm{~nm}$, Millipore Co.), freeze-dried, and stored at $4^{\circ} \mathrm{C}$ for further use. In order to obtain nanoparticles with optimal properties, different nanoformulations with varying concentrations of the polymer and stabilizer were tested, as shown in - Table 3.

\section{Size, polydispersity, and $\zeta$-potential characterization of nanoparticles}

The nano-features (size, polydispersity, and $\zeta$-potential) of curcumin-loaded PCL and MPEG-PCL nanoparticles were determined using Zetasizer Nano ZS (Malvern Instruments). For measurements, $500 \mu \mathrm{L}$ of the nanoparticle suspension was diluted with $500 \mu \mathrm{L}$ of ultrapure water. The $\zeta$-potential was determined based on the electrophoretic mobility of the nanoparticles in an aqueous solution using laser doppler velocimetry and phase analysis light scattering.

\section{Nanoparticle morphology}

Morphological characteristics of the nanoparticles were confirmed by TEM (JEOL-1400, 902A Jeol Ltd.) after negative staining with sodium phosphotungstate solution $(2 \%, w / w)$. Freshly prepared $500 \mu \mathrm{L}$ of curcumin-encapsulated nanoparticle suspension was diluted with $500 \mu \mathrm{L}$ of ultrapure water, dropped onto a copper grid (400 mesh), and used for analysis.

\section{$\mathrm{X}$-ray diffraction analysis}

XRD studies on pure curcumin, PCL, MPEG-PCL, and curcuminloaded PCL and MPEG-PCL nanoparticles were performed using an X-ray diffractometer (Philips, PW1710) with a horizontal goniometer. The samples were placed in the sample holder and scanned at a rate of $1^{\circ} \mathrm{min}^{-1}$ from $0^{\circ}$ to $60^{\circ}$.

\section{Differential scanning calorimetry}

The physical state of curcumin entrapped in the nanoparticles was characterized by differential scanning calorimetry (Universal V4.7A TA Instruments). The endothermic melting temperature of the drug (curcumin) polymer (PCL and MPEG-PCL) and curcuminloaded PCL and MPEG-PCL nanoparticles was measured by placing 5-10 $\mathrm{mg}$ of samples on aluminum pans with lids and scanning between $25^{\circ} \mathrm{C}$ and $320^{\circ} \mathrm{C}$ at the rate of $5^{\circ} \mathrm{C}$ per min under a nitrogen atmosphere.

\section{Encapsulation efficiency}

The encapsulated curcumin content was estimated by dissolving $20 \mathrm{mg}$ of curcumin-loaded nanoparticles in $2 \mathrm{~mL}$ of methanol $(\mathrm{MeOH})$ to disrupt the nanoparticle structure. Then, the sample was subjected to sonication for $5 \mathrm{~min}$ and centrifuged at 10000 rpm for $10 \mathrm{~min}$ to obtain a clear supernatant. The curcumin concentration from the sample was measured by HPLC-DAD. HPLC analyses were developed in a Merck-Hitachi LaChrom Elite HPLC apparatus equipped with an L-2450 DAD detector, L-2300 column oven, L-2130 quaternary pump, and L-2200 autosampler. Chromatographic separation was carried out on an RP C18 Lichrospher column (Merck) using water: formic acid (99:1) and 
- Table 3 Various composition of polymer, curcumin, and stabilizer used in the preparation of nanoparticles.

\begin{tabular}{|l|l|l|l|l|}
\hline $\begin{array}{l}\text { Formula- } \\
\text { tion code }\end{array}$ & $\begin{array}{l}\text { Curcumin } \\
(\mathbf{m g})\end{array}$ & $\begin{array}{l}\text { Polymer } \\
(\mathbf{m g})\end{array}$ & $\begin{array}{l}\text { Organic } \\
\text { solvent (ml) }\end{array}$ & $\begin{array}{l}\text { Stabilizer } \\
(\mathbf{\%})\end{array}$ \\
\hline PCL1 & 10 & 50 & 5 & 0.5 \\
\hline PCL2 & 10 & 100 & 5 & 0.5 \\
\hline PCL3 & 10 & 50 & 5 & 1.0 \\
\hline PCL4 & 10 & 100 & 5 & 1.0 \\
\hline PCL5 & 10 & 100 & 5 & 1.5 \\
\hline MPCL1 & 10 & 50 & 5 & 0.5 \\
\hline MPCL2 & 10 & 100 & 5 & 0.5 \\
\hline MPCL3 & 10 & 50 & 5 & 1.0 \\
\hline MPCL4 & 10 & 100 & 5 & 1.0 \\
\hline MPCL5 & 10 & 100 & 5 & 1.5 \\
\hline
\end{tabular}

$\mathrm{MeOH}$ as the mobile phases. Detection was in the $250-500 \mathrm{~nm}$ range, and chromatograms were recorded at $425 \mathrm{~nm}$. The encapsulation efficiency of the nanoformulations was determined using the following equation:

Encapsulation efficiency $=$

Amount of extract or curcumin in the nanoparticles Total amount used for preparation 100

\section{Solubility analysis}

The solubility of free curcumin and encapsulated curcumin in PBS solution ( $0.01 \mathrm{M}, \mathrm{pH} 7.4)$ was investigated. Aliquots of free curcumin and curcumin-loaded PCL and MPEG-PCL nanoparticles were added to amber vials containing PBS solution $(5 \mathrm{ml})$ in sufficient amounts to exceed the saturation point of the solvent. The vials containing the solutions were submitted to sonication for $5 \mathrm{~min}$, and placed overnight ( $12 \mathrm{~h}$ ) in a shaker, at $37^{\circ} \mathrm{C}$, in darkness. After this, solutions were centrifuged $(12000 \mathrm{~g}$ for $5 \mathrm{~min}$ ), and the supernatant was diluted and filtered for HPLC-DAD analysis for curcumin quantification. In the case of samples containing nanoparticles, the process was similar but an equal amount of $\mathrm{MeOH}$ was additionally added to the supernatant with sonication, prior to filtration, for disrupting the nanoparticles.

\section{In vitro release of curcumin}

The in vitro release studies were carried out using PBS pH 7.4 by a dialysis bag method with a molecular weight cutoff of $3500 \mathrm{Da}$. Twelve hours prior to usage, the semipermeable membrane was kept in PBS. Aliquots of $20 \mathrm{mg}$ of curcumin nanoparticles were placed in the dialysis tubes, which were then incubated in $50 \mathrm{~mL}$ of pre-warmed (at $37^{\circ} \mathrm{C}$ ) PBS, containing Tween 80 (0.5 wt\%). Samples $(1 \mathrm{~mL})$ were taken at several times from the incubation medium, and released curcumin was quantified by HPLC, as described above. After sampling, an equal volume of fresh PBS was immediately added to the incubation medium. The concentration of curcumin released from the nanoparticles was expressed as a percentage of the total curcumin present in the nanoparticles, and plotted as a function of time.

\section{Cell culture}

U251 (Human glioblastoma cell line) cells were maintained in DMEM supplemented with $1 \%$ streptomycin and $10 \% \mathrm{FBS}$, and incubated in a $\mathrm{CO}_{2}$ incubator at $37^{\circ} \mathrm{C}, 5 \% \mathrm{CO}_{2}$.

\section{Cytotoxicity assay}

The safety range and possible cytotoxic concentrations of free curcumin and loaded PCL and MPEG-PCL nanoparticles on U251 cell viability was determined using the MTT-based colorimetric assay. Cells were plated onto flat-bottom 24-well microplates at $10 \times 10^{4}$ cells per well. After incubation for $24 \mathrm{~h}$ at $37^{\circ} \mathrm{C}$, culture medium was removed and replaced with $500 \mu \mathrm{L}$ medium containing varying concentrations of empty PCL and MPEG-PCL nanoparticles, free curcumin, and curcumin-loaded PCL and MPEG-PCL nanoparticles. Additionally, $500 \mu \mathrm{L}$ of medium containing DMSO at a concentration of $30 \%$ was used as a positive control for the cytotoxic effect. After $24 \mathrm{~h}$ incubation, the medium was removed, replaced with $500 \mu \mathrm{L}$ of MTT solution and incubated for an additional $2 \mathrm{~h}$ period. The amount of MTT which was converted to formazan indicates the number of metabolic active cells. The absorbance was red at $570 \mathrm{~nm}$ in a microplate reader (Victor 1420, PerkinElmer), and the results expressed as the percentage of viable cells. Only nontoxic concentrations were used in further cells assays.

\section{Oxidative damage assay}

The neuroprotective effect of free curcumin and curcumin-loaded PCL and MPEG-PCL nanoparticles was also analyzed by t-BHPbased MTT assay. To evaluate the effect of t-BHP in U251 cells, four different concentrations were tested, with three times the incubation, to select the appropriate insult concentration and incubation period. Oxidative damage (insult) was induced with t-BHP ( $3 \mathrm{~h}, 1 \mathrm{mM}$ ) in $\mathrm{U}-251$ cells. After induction of oxidative damage, cells were treated with different concentrations of free curcumin or equivalent amounts of curcumin loaded in PCL and MPEG-PCL nanoparticles. Quercetin $(10 \mu \mathrm{M})$ was used as a positive control for neuroprotective effect against t-BHP insult. Cell viability was analyzed by the MTT assay.

\section{Cellular uptake of nanoparticles}

To investigate cellular uptake of these nanoparticles, U251 cells were seeded in 24-well microplates and allowed to attach and grow. After $24 \mathrm{~h}$, cells were incubated with $0.5 \mathrm{~mL}$ of free curcumin or curcumin-loaded PCL and MPEG-PCL nanoparticles for different time periods (2, 4, and $24 \mathrm{~h}$ ). Cells were washed with PBS and fixed with paraformaldehyde. After fixation, the cells were incubated with Hoechst 33342 for nuclei staining and analyzed under a fluorescent microscope (Olympus IX71).

\section{In vivo distribution of nanoparticles in zebra fish embryos}

The in vivo distribution of nanoparticles was assessed via zebra fish embryo toxicity (ZET) testing. For zebra fish egg production, a male-biased sex:ratio group of parental individuals was housed 
in a cage with a net bottom covered with glass marbles within a 30-L aquarium under controlled physicochemical conditions (photoperiod of $14: 10 \mathrm{~h}$, light: dark; temperature of $\left.28 \pm 1^{\circ} \mathrm{C}\right)$. The breeding protocol was done according to Nasiadaka and Clark [26]. Two hours post-fertilization (hpf), zygotes were separated from the unviable eggs taking into account the absence of optical transparency in the former ones under with a stereomicroscope (Nikon Eclipse TS 100). The selected eggs were washed twice with autoclaved freshwater and randomly allocated into 24-well microplates for continuous waterborne incubation with free curcumin, and empty and curcumin-loaded MPEG-PCL and PCL nanoparticles, for $80 \mathrm{hpf}$. All embryos used in the experiments were derived from the same spawn of eggs, which presented a fertilization rate higher than $90 \%$. Ten embryos were incubated per well in a final volume of $2 \mathrm{~mL}$. Each condition was tested in quadruplicate, including the control. The incubation media was renewed every $24 \mathrm{~h}$ and dead embryos were removed three times every $24 \mathrm{~h}$ to circumvent cross-contamination. Two different concentrations $(0.25$ and $1 \mu \mathrm{g} / \mathrm{mL})$ were tested. To ensure the optimal incubation temperature of the zebra fish embryos, all the test conditions were preheated to $28 \pm 1{ }^{\circ} \mathrm{C}$. The embryonic development of zebra fish was followed until $80 \mathrm{hpf}$ and recorded under a fluorescent microscope (Olympus IX71).

\section{Statistical analysis}

All parameters were statistically analyzed using the software GraphPad 5 (Prism). Data are expressed as the mean \pm S. E. M. of the indicated number of experiments. Mean differences were statistically evaluated using the unpaired two-tailed Student's t-test. A difference of $p<0.05$ was considered significant.

\section{Conflict of Interest}

The authors declare no conflict of interest.

\section{References}

[1] Gong C, Deng S, Wu Q, Xiang M, Wei X, Li L, Gao X, Wang B, Sun L, Chen Y, Li Y, Liu L, Qian Z, Wei Y. Improving antiangiogenesis and anti-tumor activity of curcumin by biodegradable polymeric micelles. Biomaterials 2013; 34: 1413-1432

[2] Gregory M, Sarmento B, Duarte S, Martins JA, Silva C, Coutinho O, Dias ACP. Curcumin loaded MPEG-PCL di-block copolymer nanoparticles protect glioma cells from oxidative damage. Planta Med 2014; 80: 14671468

[3] Egan ME, Pearson M, Weiner SA, Rajendran V, Rubin D, Glockner-Pagel ], Canny S, Du K, Lukacs GL, Caplan MJ. Curcumin, a major constituent of turmeric, corrects cystic fibrosis defects. Science 2004; 304: 600-602

[4] Jagetia GC, Aggarwal BB. "Spicing up" of the immune system by curcumin. J Clin Immunol 2007; 27: 19-35

[5] Gong CY, Wei XW, Wang XH, Wang Y], Guo G, Mao YQ, Luo F, Qian ZY. Biodegradable self-assembled PEG-PCL-PEG micelles for hydrophobic honokiol delivery: I. Preparation and characterization. Nanotechnology 2010; 21: 215103-215111

[6] Li L, Braiteh FS, Kurzrock R. Liposome-encapsulated curcumin: in vitro and in vivo effects on proliferation, apoptosis, signaling, and angiogenesis. Cancer 2005; 104: 1322-1331

[7] Ray B, Bisht S, Maitra A, Lahiri DK. Neuroprotective and neurorescue effects of a novel polymeric nanoparticle formulation of curcumin (Nano-
Curc) in the neuronal cell culture and animal model: implications for Alzheimer's disease. J Alzheimers Dis 2011; 23: 61-77

[8] Dhawan S, Kapil R, Singh B. Formulation development and systematic optimization of solid lipid nanoparticles of quercetin for improved brain delivery. J Pharm Pharmacol 2011; 63: 342-351

[9] Hu Y, Jiang X, Ding Y, Zhang L, Yang C, Zhang J, Chen J, Yang Y. Preparation and drug release behaviors of nimodipine-loaded poly(caprolactone)-poly(ethylene oxide)-polylactide amphiphilic copolymer nanoparticles. Biomaterials 2003; 24: 2395-2404

[10] Shao JF, Zheng DH, jiang ZF, Xu HE, Hu Y, Li XL, Lu XW. Curcumin delivery by methoxy polyethylene glycol-poly(caprolactone) nanoparticles inhibits the growth of C6 glioma cells. Acta Biochim Biophys Sin (Shanghai) 2011; 43: 267-274

[11] Yuan M, Wang Y, Li X, Xiong C, Deng X. Polymerization of lactides and lactones. 10. Synthesis, characterization, and application of amino-terminated poly(ethylene glycol)-co-poly( $\varepsilon$-caprolactone) block copolymer. Macromolecules 2000; 33: 1613-1617

[12] Peng W, Jiang XY, Zhu Y, Omari-Siaw E, Deng WW, Yu JN, Xu XM, Zhang WM. Oral delivery of capsaicin using MPEG-PCL nanoparticles. Acta Pharmacol Sin 2015; 36: 139-148

[13] Wang K, Liu TT, Lin R, Liu B, Yang GD, Bu X, Wang WR, Zhang PP, Zhou L, Zhang JY. Preparation and in vitro release of buccal tablets of naringeninloaded MPEG-PCL nanoparticles. RSC Adv 2014; 4: 33672-33679

[14] Jokerst JV, Lobovkina T, Zare RN, Gambhir SS. Nanoparticle PEGylation for imaging and therapy. Nanomedicine (Lond) 2011; 6: 715-728

[15] Vij N, Min T, Marasigan R, Belcher CN, Mazur S, Ding H, Yong KT, Roy I. Development of PEGylated PLGA nanoparticle for controlled and sustained drug delivery in cystic fibrosis. J Nanobiotechnology 2010; 8: 22-40

[16] Savjani KT, Gajjar AK, Savjani JK. Drug solubility: importance and enhancement techniques. ISRN Pharm 2012; 2012: 195727

[17] Ghosh A, Banerjee T, Bhandary S, Surolia A. Formulation of nanotized curcumin and demonstration of its antimalarial efficacy. Int J Nanomedicine 2014; 9: 5373-5387

[18] Sylvester PW. Optimization of the tetrazolium dye (MTT) colorimetric assay for cellular growth and viability. Methods Mol Biol 2011; 716: 157168

[19] Ucisik MH, Kupcu S, Schuster B, Sleytr UB. Characterization of CurcuEmulsomes: nanoformulation for enhanced solubility and delivery of curcumin. J Nanobiotechnology 2013; 11: 37

[20] Liu J, Xu L, Liu C, Zhang D, Wang S, Deng Z, Lou W, Xu H, Bai Q, Ma J. Preparation and characterization of cationic curcumin nanoparticles for improvement of cellular uptake. Carbohydr Polym 2012; 90: 16-22

[21] Mohanty C, Sahoo SK. The in vitro stability and in vivo pharmacokinetics of curcumin prepared as an aqueous nanoparticulate formulation. Biomaterials 2010; 31: 6597-6611

[22] Gong C, Deng S, Xiang M, Wei X, Li L, Gao X, Wang B, Sun L, Chen Y, Li Y, Qian Z, Wei Y. Improving antiangiogenesis and anti-tumor activity of curcumin by biodegradable polymeric micelles. Biomaterials 2013; 34: $1413-1432$

[23] Liu X, Ye M, An C, Pan L, Ji L. The effect of cationic albumin-conjugated PEGylated tanshinone IIA nanoparticles on neuronal signal pathways and neuroprotection in cerebral ischemia. Biomaterials 2013; 34: 68936905

[24] Ganesan P, Ko HM, Kim IS, Choi DK. Recent trends in the development of nanophytobioactive compounds and delivery systems for their possible role in reducing oxidative stress in Parkinson's disease models. Int J Nanomedicine 2015; 10: 6757-6772

[25] Batrakova EV, Kabanov AV. Pluronic block copolymers: evolution of drug delivery concept from inert nanocarriers to biological response modifiers. J Control Release 2008; 130: 98-106

[26] Nasiadka A, Clark MD. Zebrafish breeding in the laboratory environment. ILAR J 2012; 53: 161-168 